Coaching und Gesellschaft 
Robert Wegener - Michael Loebbert Agnès Fritze

Herausgeber

\section{Coaching und Gesellschaft}

Forschung und Praxis im Dialog

照 Springer 
Herausgeber

Robert Wegener

Institut Berat., Coach. u. Soz.man.

Fachhochschule Nordwestschweiz

Olten, Schweiz

Agnès Fritze

Institut Berat., Coach. u. Soz.man.

Fachhochschule Nordwestschweiz

Olten, Schweiz
Michael Loebbert

Institut Berat., Coach. u. Soz.man.

Fachhochschule Nordwestschweiz

Olten, Schweiz

ISBN 978-3-658-09635-9

ISBN 978-3-658-09636-6 (eBook)

DOI 10.1007/978-3-658-09636-6

Die Deutsche Nationalbibliothek verzeichnet diese Publikation in der Deutschen Nationalbibliografie; detaillierte bibliografische Daten sind im Internet über http://dnb.d-nb.de abrufbar.

Springer

(C) Springer Fachmedien Wiesbaden 2016

Das Werk einschließlich aller seiner Teile ist urheberrechtlich geschützt. Jede Verwertung, die nicht ausdrücklich vom Urheberrechtsgesetz zugelassen ist, bedarf der vorherigen Zustimmung des Verlags. Das gilt insbesondere für Vervielfältigungen, Bearbeitungen, Übersetzungen, Mikroverfilmungen und die Einspeicherung und Verarbeitung in elektronischen Systemen.

Die Wiedergabe von Gebrauchsnamen, Handelsnamen, Warenbezeichnungen usw. in diesem Werk berechtigt auch ohne besondere Kennzeichnung nicht zu der Annahme, dass solche Namen im Sinne der Warenzeichen- und Markenschutz-Gesetzgebung als frei zu betrachten wären und daher von jedermann benutzt werden dürften.

Der Verlag, die Autoren und die Herausgeber gehen davon aus, dass die Angaben und Informationen in diesem Werk zum Zeitpunkt der Veröffentlichung vollständig und korrekt sind. Weder der Verlag noch die Autoren oder die Herausgeber übernehmen, ausdrücklich oder implizit, Gewähr für den Inhalt des Werkes, etwaige Fehler oder Äußerungen.

Gedruckt auf säurefreiem und chlorfrei gebleichtem Papier

Springer Fachmedien Wiesbaden ist Teil von Springer Nature

Die eingetragene Gesellschaft ist Springer Fachmedien Wiesbaden GmbH 


\section{Inhaltsverzeichnis}

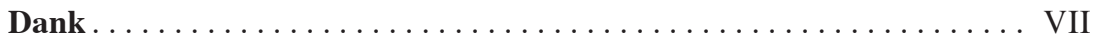

Geleitwort von Siegfried Greif. $\ldots \ldots \ldots \ldots \ldots \ldots \ldots \ldots \ldots \ldots \ldots \ldots$

Coaching und Gesellschaft - Einleitende und einladende Gedanken .... 1 Robert Wegener, Michael Loebbert und Agnès Fritze

Unruhe stiften: Coaching als Medium und Form ............. 9 Dirk Baecker

Third Generation Coaching -

Ein Plädoyer für die Weiterentwicklung einer Dialogform . . . . . . . 25

Reinhard Stelter

Die Bewertung von Coaching-Prozessen als ethische Herausforderung. . 49 Harald Geißler

Beratung und soziale Selbstreflexivität -

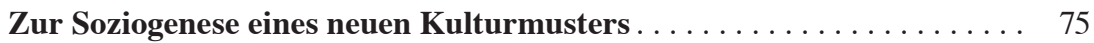

Beate Fietze

Coaching im Kontext der Organisation ................. 93

Michael Loebbert, Louis Klein und Markus Rettich

Gott schuf den Menschen, und der formt sich selbst:

Coaching neu gedacht. . .

Jesus Hernandez Aristu 


\section{Zeigt Führungskräfte-Coaching Wirkung?}

Und wenn ja, wie wirkt es?

Nadine Page und Erik de Haan

Coaching als Chance oder Zwang:

ein bilanzierendes Schlusswort . .

Marianne Hänseler 
Der vorliegende Band »Coaching und Gesellschaft. Forschung und Praxis im Dia$\log$ « besteht aus Beiträgen zum dritten internationalen Coachingkongress »Coaching meets Research ... Coaching in der Gesellschaft von morgen«, der von der Hochschule für Soziale Arbeit der Fachhochschule Nordwestschweiz FHNW organisiert und im Juni 2014 in Olten/Schweiz durchgeführt wurde. Für die Bereitschaft, sich konzeptionell an den Anforderungen der Herausgebenden zu orientieren, danken wir den Autorinnen und Autoren ganz herzlich.

Wir danken ferner den folgenden Partnern für ihre Unterstützung, die den Kongress und diese Publikation erst ermöglicht hat:

\section{Hauptsponsoren}

- Berufsverband für Coaching, Supervision und Organisationsberatung BSO Schweiz

- Böning-Consult GmbH, Frankfurt

- Coachingzentrum Olten

- European Mentoring \& Coaching Council Schweiz

- Föderation der Schweizer Psychologinnen und Psychologen

- International Coach Federation Schweiz

- Karlsruher Institut für Coaching in Kooperation mit Prof. Dr. Geißler

\section{Hochschulpartner}

- Ashridge Business School

- Coaching Psychology Unit, NEXS, University of Copenhagen 
- Hochschule für Wirtschaft FHNW

- Oxford Brookes University

- Zürcher Hochschule für Angewandte Wissenschaften Departement Angewandte Psychologie

\section{Medienpartner}

- Coaching-Magazin

- Magazin Training

- MWOnline

- Organisationsberatung, Supervision, Coaching

- Springer

\section{Unternehmenspartner}

- CoachingSpaces

- Curaviva Weiterbildung

- Institut für Systemische Impulse, Entwicklung und Führung

- MDOb an der Management School der Universität Kassel

- permitto Schweiz AG

- Trigon Entwicklungsberatung

\section{Verbandspartner $(*$ Mitglied des Roundtable der Coaching- Verbände)}

- Association des Psychologues du Travail et des Organisations en Suisse romande

- Deutscher Bundesverband Coaching e. V.*

- Deutscher Coaching Verband e. V.*

- Deutsche Gesellschaft für Coaching e. V.*

- Deutsche Gesellschaft für Supervision e. V.*

- Deutsche Gesellschaft für Systemische Therapie, Beratung und Familientherapie e. V.

- European Association for Supervision and Coaching e. V**

- European Mentoring \& Coaching Council Deutschland*

- Fachgruppe Wien Unternehmensberatung und Informationstechnologie 
- Österreichische Vereinigung für Supervision

- Swiss Coaching Association

Ein herzlicher Dank geht an Cheryl Meyer, die als unterstützende Fachkraft zur Entstehung dieses Buches maßgeblich beigetragen hat. Ein großer Dank geht auch an Christoph Gassmann, der das Manuskript mit kritischem Blick, inhaltlichem Flair und großer Sorgfalt lektoriert und korrigiert hat. Nicht zuletzt danken wir dem Springer Verlag und insbesondere Frau Eva Brechtel-Wahl und Frau Sonja Trautwein für die kompetente Begleitung. 


\section{Geleitwort von Siegfried Greif}

Coaching bedient eine zunehmende Individualisierung in den westlichen Gesellschaften. Selbstbewusste Individuen holen sich Coaches als Reflexions- und Diskurspartnerinnen und -partner für ihre persönlichen Zielsetzungen. Unternehmen (an)erkennen die interindividuellen Unterschiede ihrer Führungskräfte und Mitarbeitenden und ermöglichen durch Einzelcoaching individuell angepasste, selbstgesteuerte Verhaltensänderungen. Wenn Coaches die Individualisierung in der Gesellschaft jedoch lediglich als willkommene Nachfrage für das eigene Geschäft nutzen und dabei die beteiligten komplexen gesellschaftlichen Entwicklungen und Zusammenhänge unberücksichtigt lassen, bleibt ihr Coaching oberflächlich und verkürzt.

Die Klientinnen und Klienten stellen oft selbst Bezüge zu aktuellen gesellschaftlichen Entwicklungen her, wenn sie über eine schwierige wirtschaftliche Situation ihres Unternehmens und ihrer beruflichen Perspektive sprechen, wenn sie organisationale Veränderungen mit ungewissen Risiken thematisieren, wenn sie über Zeitdruck und Mehrarbeit klagen, die sie als Arbeit und Belastung mit nach Hause in ihre Familie nehmen, und wenn sie fragen, woran sie sich orientieren können, wenn sich frühere Traditionen, Regeln und Normen auflösen und wenn sie bei der Suche nach dem Sinn ihres Lebens individuell auf sich selbst zurückgeworfen werden. Soziologinnen und Psychologen analysieren diese gesellschaftlichen und individuellen Entwicklungen unterschiedlich, aber aufeinander beziehbar als Konsequenz des globalen Wettbewerbs, der komplexen oder hyperkomplexen posttraditionellen Risikogesellschaft und der Beschleunigung ihrer Veränderungsdynamiken und ihrer Unvorhersehbarkeit. Coaching ist ein wichtiger Ort, der es ermöglicht, über diese gesellschaftlichen Entwicklungen und über sich selbst ergebnisorientiert zu reflektieren. Mehrere Beiträge stellen in Anlehnung an den Soziologen Anthony Giddens die grundlegende Bedeutung der Selbstreflexivität für komplexe Gesellschaften heraus. 
Die Autorinnen und Autoren diese Buchs nehmen verschiedene Facetten des Themas »Coaching und Gesellschaft« auf und vertiefen sie. Die aufgeworfenen Fragen werden auf der Grundlage unterschiedlicher Wissenschaftsrichtungen, Fachdisziplinen und Einzeltheorien analysiert und diskutiert. Das Buch enthält einen Diskursbeitrag mehrerer Autoren und eine Zusammenschau als Abschluss, in der solche Unterschiede angesprochen werden. Das Thema »Coaching und Gesellschaft « ist ein außerordentlich weites Feld. Das Buch ist ein anspruchsvoller und interessanter Anfang des für die Coaching-Profession grundlegenden Diskurses. Es ist wichtig, es zu lesen und sich an diesem Diskurs zu beteiligen - wichtig, um für die eigene gesellschaftliche Rolle als Coach und im Interesse der Klientinnen und Klienten tiefere Einsichten in die komplexen Entwicklungszusammenhänge zu gewinnen.

Mai 2016

Siegfried Greif 